\section{The innate sensor ZBP1-IRF3 axis regulates cell proliferation in multiple myeloma}

\author{
Kanagaraju Ponnusamy, ${ }^{1}$ Maria Myrsini Tzioni, ${ }^{1}$ Murshida Begum, ${ }^{1}$ \\ Mark E. Robinson, ${ }^{1}$ Valentina S. Caputo, ${ }^{1}$ Alexia Katsarou, ${ }^{1,2}$ \\ Nikolaos Trasanidis, ${ }^{1}$ Xiaolin Xiao, ${ }^{1}$ loannis V. Kostopoulos, ${ }^{1,3}$ \\ Deena Iskander, ${ }^{1,2}$ Irene Roberts, ${ }^{4}$ Pritesh Trivedi, ${ }^{5}$ Holger W. Auner, ${ }^{1,2}$ \\ Kikkeri Naresh, ${ }^{5}$ Aristeidis Chaidos ${ }^{1,2}$ and Anastasios Karadimitris ${ }^{1,2}$
}

${ }^{1}$ Hugh \& Josseline Langmuir Centre for Myeloma Research, Centre for Haematology, Department of Immunology \& Inflammation, Imperial College London, London, UK; ${ }^{2}$ Department of Haematology, Hammersmith Hospital, Imperial College Healthcare NHS Foundation Trust, London, UK; ${ }^{3}$ Section of Animal and Human Physiology, National and Kapodestrian University of Athens, Department of Biology, School of Science, Athens, Greece; ${ }^{4}$ Department of Paediatrics and MRC Molecular Haematology Unit, Weatherall Institute of Molecular Medicine, University of Oxford and BRC Blood Theme, NIHR Oxford Biomedical Centre, Oxford, UK and ${ }^{5}$ Department of Cellular \& Molecular Pathology, Northwest London Pathology, Imperial College Healthcare NHS Trust, London, UK

\section{ABSTRACT}

M ultiple myeloma is a malignancy of plasma cells initiated and driven by primary and secondary genetic events. However, myeloma plasma cell survival and proliferation might be sustained by non-genetic drivers. Z-DNA-binding protein 1 (ZBP1; also known as DAI) is an interferon-inducible, Z-nucleic acid sensor that triggers RIPK3-MLKL-mediated necroptosis in mice. ZBP1 also interacts with TBK1 and the transcription factor IRF3 but the function of this interaction is unclear, and the role of the ZBP1-IRF3 axis in cancer is not known. Here we show that ZBP1 is selectively expressed in late B-cell development in both human and murine cells and it is required for optimal T-cell-dependent humoral immune responses. In myeloma plasma cells, the interaction of constitutively expressed ZBP1 with TBK1 and IRF3 results in IRF3 phosphorylation. IRF3 directly binds and activates cell cycle genes, in part through co-operation with the plasma cell lineage-defining transcription factor IRF4, thereby promoting myeloma cell proliferation. This generates a novel, potentially therapeutically targetable and relatively selective myeloma cell addiction to the ZBP1-IRF3 axis. Our data also show a noncanonical function of constitutive ZBP1 in human cells and expand our knowledge of the role of cellular immune sensors in cancer biology.

\section{Introduction}

Multiple myeloma (MM) is a common incurable blood cancer of the bone marrow plasma cells $(\mathrm{PC})$, the immunoglobulin-secreting terminally differentiated $\mathrm{B}$ lineage cells. ${ }^{1-3}$ Primary and secondary somatic genetic events comprising copy number and single nucleotide variants shape a genomic landscape of extensive, in time and space, genetic heterogeneity and diversification rendering targeted therapies for MM a challenging task. ${ }^{1-3}$ In this regard, there is a need for identification of biological pathways that are involved in myelomagenesis independently of genetic status.

Previous studies of murine late $B$ lineage development identified $Z B P 1$ as one of the genes that define the transcriptional signature of follicular $B$-cell transition to plasmablasts and mature PC. ${ }^{4} \mathrm{ZBP} 1$ is an inducible cellular DNA/RNA sensor with two $Z \alpha$ domains that bind pathogen-derived or cellular Z-DNA ${ }^{5,6}$ or Z-RNA. ${ }^{7,8} Z \alpha-$ dependent nucleic acid sensing induces a RHIM-RHIM domain interaction of ZBP1 with receptor-interacting protein kinase 3 (RIPK3) which ultimately triggers mixed lineage kinase domain like pseudokinase (MLKL)-mediated necroptosis. ${ }^{7-10}$ This process is counteracted by RIPK1, preventing for example ZBP1-dependent cell death and inflammation in the developing skin. ${ }^{11-13}$
Haematologica 2022

Volume 107(3):721-732

\section{Correspondence:}

ANASTASIOS KARADIMITRIS

a.karadimitris@imperial.ac.uk

Received: October 20, 2020.

Accepted: February 2, 2021.

Pre-published: February 18, 2021.

https://doi.org/10.3324/haematol.2020.274480

(C2022 Ferrata Storti Foundation

Material published in Haematologica is covered by copyright. All rights are reserved to the Ferrata Storti Foundation. Use of published material is allowed under the following terms and conditions:

https://creativecommons.org/licenses/by-nc/4.0/legalcode. Copies of published material are allowed for personal or internal use. Sharing published material for non-commercial purposes is subject to the following conditions:

https://creativecommons.org/licenses/by-nc/4.0/legalcode, sect. 3. Reproducing and sharing published material for commercial purposes is not allowed without permission in writing from the publisher. 
In addition, like other nucleic acid sensors, such as cGas/STING, ZBP1 has been shown to associate with serine/threonine kinase TBK1 with subsequent phosphorylation of the transcription factor IRF3 (pIRF3). Activation of STING and other nucleic acid sensors triggers translocation of pIRF3 to the nucleus where it directly activates transcription of interferon (IFN) type I response genes, ${ }^{14-16}$ however, this has been disputed in the case of $Z B P 1^{17,18}$ Therefore, the role of ZBP1-TBK1-IRF3 in innate sensing or in the context of cancer remains unclear. Nevertheless, it is clear that ZBP1 itself is an IFN-inducible gene as its expression is induced by type I and II IFN which leads to RIPK3-dependent necroptosis ${ }^{7,13,19}$ and IFNAR1 ${ }^{-/}$cells fail to upregulate virus-induced ZBP1 expression. ${ }^{20}$ Since recent work demonstrated an active type I IFN versus proliferative transcriptional signature prevailing in early diagnosis versus relapsed patients' myeloma PC or myeloma cell lines, respectively, ${ }^{21}$ we hypothesized that ZBP1 might regulate myeloma PC biology.

\section{Methods}

\section{Cell culture, primary samples}

U266 and NCI-H929 (Deutsche Sammlung von Mikroorganismen und Zellkulturen, Germany), MM.1R and MM.1S (American Type Culture Collection, Manassas, VA, USA), and HeLa, HEK293T, DU145, MCF7, HCC95, SF295, LNCAP, K562, Jurkat and C1R cells were cultured in either $10 \%$ fetal bovine serum, RPMI-1640 or Dulbecco modified Eagle medium. Primary myeloma cells were collected under ethical committee approval (REC n. 11/H0308/9) and cultured with IL-6 (10 ng/mL).

\section{Cell cycle, proliferation and apoptosis}

Cell cycle status was assessed using flow cytometry after staining cells with $10 \mu \mathrm{M}$ Hoechst-33342 in RPMI-1640 medium for 1 h. The cytostatic effects of constitutive or inducible shRNA-transduced cells were measured as shown in Online Supplementary Figure S3C. Annexin V (BioLegend) staining was performed according to the manufacturer's instructions.

\section{Subcutaneous tumor model, immunization}

The subcutaneous tumor study was performed in NOD/SCID $\gamma$ mice (license PPL70/8586), by subcutaneously injecting $10^{7}$ doxycycline-inducible shRNA- transduced cells with Matrigel (Corning). Tumor volume was calculated by the formula $\left(1 / 2\left[\right.\right.$ length $\left.\left.\times w_{i d t h}^{2}\right]\right)$. Animals were administered $100 \mu \mathrm{g} / \mathrm{mL}$ doxycycline in drinking water and $0.2 \mathrm{mg} / \mathrm{kg}$ intraperitoneally.

Zbp $1^{1 /}$ animals ${ }^{18}$ were obtained from Manolis Pasparakis, Institute of Genetics (Cologne, Germany). Ten- to 12-week-old littermates were immunized by intraperitoneal injection of $4 \mathrm{mg} / \mathrm{kg}$ NP-KLH (Santacruz Biotech) with Alum Adjuvant (Thermoscientific) at a 3:1 ratio followed by a booster dose of NPKLH on day 4. Serum NP-KLH-specific antibodies were measured.

\section{Quantitative polymerase chain reaction analysis, RNA-sequencing}

Total RNA was isolated using a Nucleospin RNA kit (MachereyNagel) followed by cDNA synthesis using a RevertAid cDNA synthesis kit (Thermoscientific). Indicated cDNA were quantified using the primers shown in the Online Supplementary Methods. Poly(A)-tail mRNA was isolated, from total RNA of fluorescenceactivated cell sorting (FACS)-purified green fluorescent protein $(\mathrm{GFP})^{+}$cells with either scrambled or shRNA targeting ZBP1 or IRF3 on day 4 after transduction when $>80 \%$ knockdown was achieved, using the NEBNext poly(A) mRNA Magnetic Isolation Module, and libraries were prepared using NEBNext Ultra II RNA library prep kits (New England Biolabs). A 2 nM DNA library (350$400 \mathrm{bp}$ ) was sequenced using Illumina HiSeq 2500 (for shZBP1) or NextSeq500 (for shIRF3) for paired-end 150 bp reads.

\section{Chromatin immunoprecipitation (ChIP)-sequencing, ChIP-re-ChIP}

MM.1S cells were cross-linked with $1 \%$ formaldehyde and lysed with hypotonic lysis buffer followed by nuclear lysis buffer. The lysate was sonicated to shear the chromatin up to $500 \mathrm{bp}$ followed by pre-clear with protein A/G magnetic beads and immunoprecipitation with IRF3 antibody or equivalent isotype control. ChIP DNA was collected with AMPure XP-beads after reverse cross-linking. ChIP DNA (1 ng) was used for library preparation with an NEBNext kit. The 2 nM DNA library (400-500 bp) was sequenced using Illumina NextSeq500. For ChIP-re-ChIP, pulled chromatin was eluted in 1\% sodium dodecylsulfate (SDS) and diluted 10 times with elution buffer and repeated re-ChIP with the appropriate antibody. The detailed protocols are provided in the Online Supplementary Methods.

\section{Co-immunoprecipitation, immunoblotting}

Co-immunoprecipitation was performed as described previous$1 y^{12}$ with $5 \%$ anti-strep-tagII-magnetic bead slurry (IBA) or $2 \mu \mathrm{g}$ anti-ZBP1 or anti-V5-Tag or anti-IRF3 or their equivalent isotype control antibodies conjugated with protein $\mathrm{A} / \mathrm{G}$ magnetic beads. For immunoblotting, total cell proteins denatured in $1 \mathrm{x}$ LDS Sample Buffer (ThermoFisher Scientific), were resolved in $10 \%$ SDS polyacrylamide gel electrophoresis and transferred to polyvinylidene fluoride membranes followed by probing with appropriate antibodies shown in the Online Supplementary Methods.

\section{Data analysis}

The details of the data analysis are provided in the Online Supplementary Methods. In brief, RNA-sequencing and ChIPsequencing reads were aligned using STAR or Salmon and BWA $M E M,{ }^{22,23}$ respectively, to the GRCh38 genome. Differential expression analysis was performed using DESeq2 or limmavoom..$^{22,24,25}$ ChIP-sequencing peaks were called with MACS2 ${ }^{26}$ and tracks with Deeptools ${ }^{27}$ and visualized in IGV. ${ }^{28}$ Homer was used for Motif analysis ${ }^{29}$ and the BETA-plus package ${ }^{30}$ to integrate RNA-sequencing and ChIP-sequencing data. Gene set enrichment analysis (GSEA) ${ }^{31}$ and the Enrichr web tool ${ }^{32}$ were used for pathway analysis.

\section{Data availability}

All next-generation sequencing data for RNA-sequencing and ChIP-sequencing experiments can be accessed via the Gene Expression Omnibus (GSE163497).

\section{Additional methods}

The details of shRNA sequences, cloning strategies, reagents, antibodies and additional methodologies are provided in the Online Supplementary Methods.

\section{Results}

\section{Restricted and constitutive expression of ZBP1 \\ in normal and myeloma plasma cells}

By searching for genes selectively expressed in $M M$ we identified ZBP1 as highly and selectively expressed in MM cell lines (MMCL) but not in other cancer cells (CCLE 
A

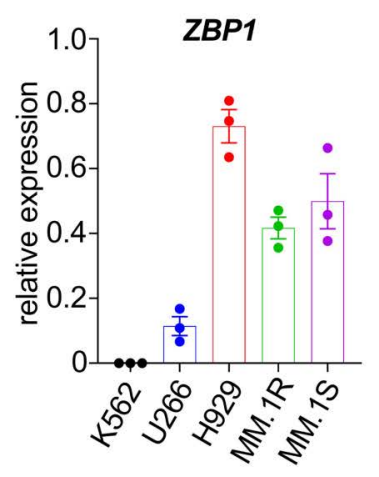

D

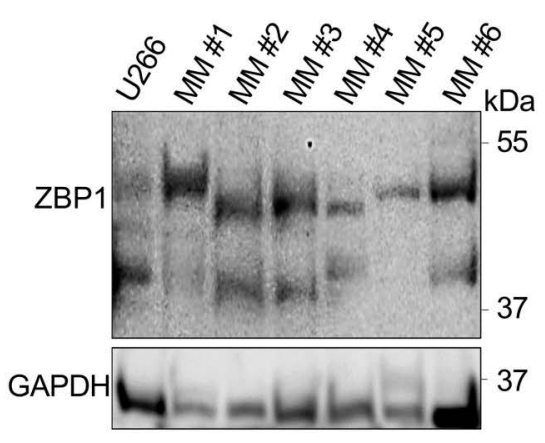

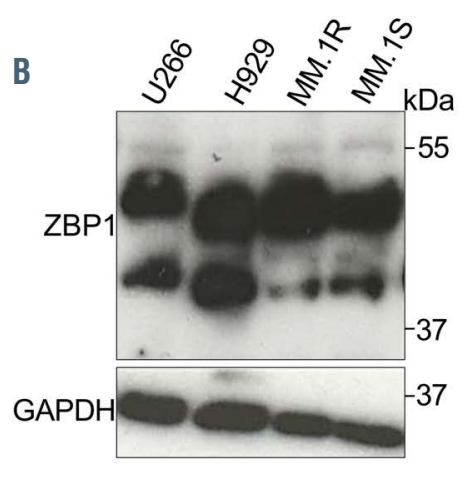

C.

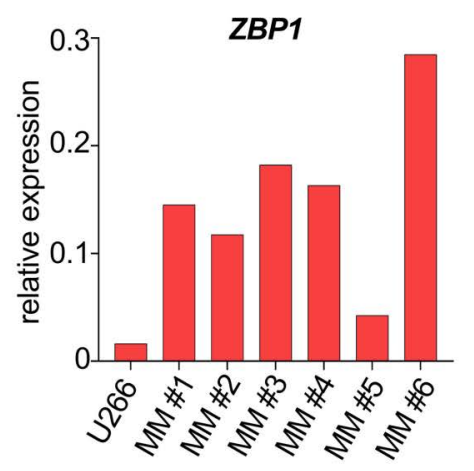

$\mathrm{E}$

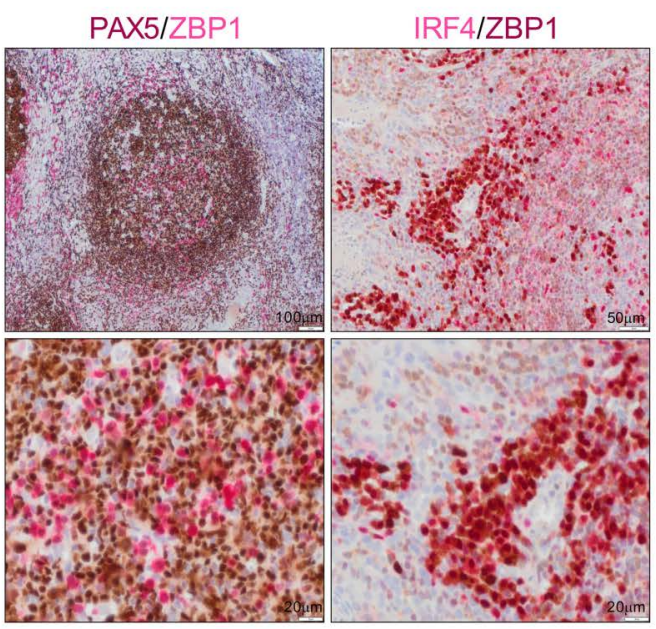

$\mathbf{F}$

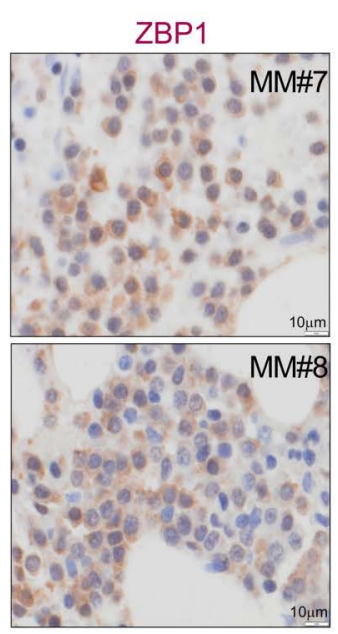

Figure 1. Restricted and constitutive ZBP1 expression in normal and myeloma plasma cells. (A) ZBP1 mRNA expression as assessed in four multiple myeloma cell lines (MMCL) and in the erythromyeloid cell line K562 by quantitative polymerase chain reaction ( $n=3$; data shown as mean \pm Standard error of mean). (B) ZBP1 expression in indicated MMCL as assessed by immunoblotting using GAPDH as the loading control. Two main isoforms $\sim 48$ and $\sim 40 \mathrm{kDa}$ are detected in MMCL. (C,

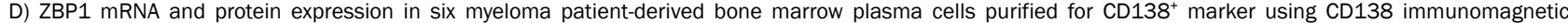
microbeads, and U266 MMCL shown as control. (E) Immunohistochemistry on paraffin-embedded tonsil tissue section. The panel on the left depicts low and high magnification of a germinal center in which expression of PAX5 and ZBP1 is mutually exclusive in most parts indicating ZBP1 expression in plasma cells that are negative for PAX5. The panel on the right depicts subepithelial tonsillar plasma cells showing co-expression of IRF4 (MUM.1) and ZBP1. (F) Immunohistochemistry for ZBP1 expression in paraffin-embedded bone marrow tissue sections from two MM patients.

dataset) (Online Supplementary Figure $S 1 A, B)$. While there is no difference in ZBP1 expression between normal $\mathrm{PC}$ and the whole cohort of myeloma PC, ZBP1 expression is significantly higher in the hyperdiploid subgroup of myeloma PC (Arkansas GSE4581 microarray dataset) (Online Supplementary Figure S1C). ZBP1 is universally expressed in primary myeloma PC (MMRF CoMMpass dataset; $\mathrm{n}=767$ patients) (Online Supplementary Figure S1D) at similar levels as the PC-defining transcription factor PRDM1 (BLIMP1). In the human $\mathrm{B}$-cell lineage, $Z B P 1$ is expressed at a very low level in germinal center $B$ cells (GCB) and a moderate level in naive and memory B cells, but $\mathrm{PC}$ show by far the highest expression (Blueprint DCC Portal data) (Online Supplementary Figure $S 1 E$ ). We confirmed expression of ZBP1 mRNA and protein in MMCL (Figure 1A, B) and also in primary myeloma PC (Figure 1C, D) but not in other hematopoietic or epithelial cancer cells (Online Supplementary Figure S1F, G), normal blood lineage cells (Online Supplementary Figure $S 1 H, I$ ) or healthy nonhematopoietic tissues (Online Supplementary Figure S1J).
Immunohistochemistry of human tonsils and lymph nodes showed expression of ZBP1 primarily in a group of PAX5-IRF4+ GCB cells i.e., those committed to PC differentiation ${ }^{33,34}$ and in interfollicular and subepithelial IRF4 ${ }^{+}$ PC, with low-level expression in mantle zone B cells (Figure $1 \mathrm{E}$ and Online Supplementary Figure $S 1 K, L$ ). In bone marrow, expression of $Z B P 1$ is mostly restricted to myelo$\mathrm{ma}$ and normal PC and is not present in other blood lineage cells (Figure $1 \mathrm{~F}$ and Online Supplementary Figure $S 1 K, M)$. These results show constitutive and restricted expression of $Z B P 1$ in myeloma $\mathrm{PC}$ as well as in late GCB cells and normal PC.

\section{ZBP1 is required for optimal T-cell-dependent humoral immune responses in mice}

Consistent with a conserved expression pattern, a previous transcriptome analysis of murine B-cell development identified $Z b p 1$ as one of the signature genes that define transition from follicular B cells to plasmablasts and mature $\mathrm{PC}^{4}$ (Online Supplementary Figure S2). To determine 
A

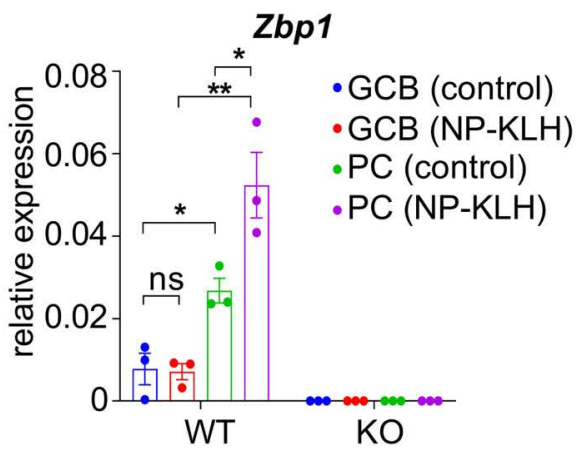

B

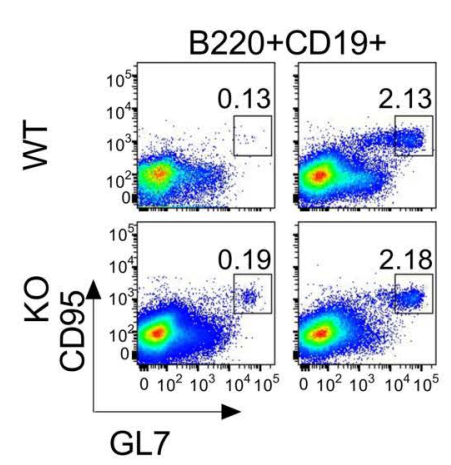

C

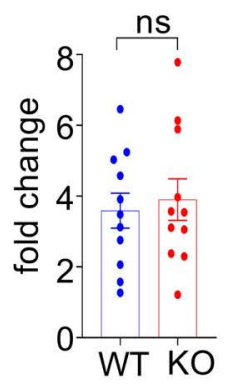

D

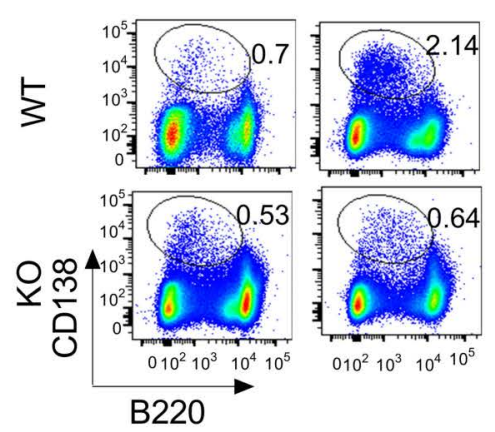

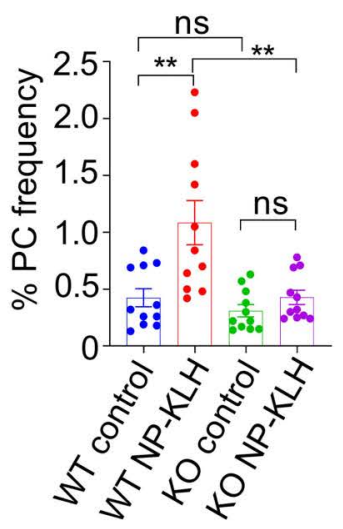

E

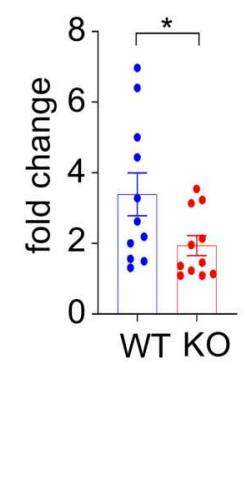

F

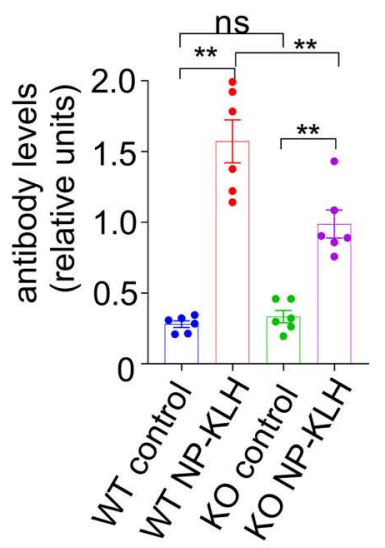

G

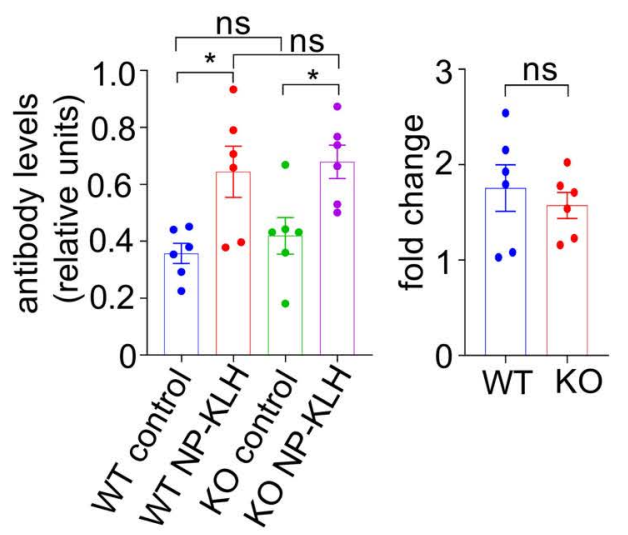

Figure 2. ZBP1 is required for optimal T cell-dependent humoral immune responses in mice. (A) Zbp1 mRNA levels as assessed by quantitative polymerase chain reaction in FACS-purified splenic germinal center $\mathrm{B}(\mathrm{GCB})$ cells $\left(\mathrm{B} 22 \mathrm{O}^{+} \mathrm{CD} 19^{+} \mathrm{GL} 7^{+} \mathrm{CD} 95^{+}\right)$and plasma cells $(\mathrm{PC})\left(\mathrm{B} 22 \mathrm{O}^{\mathrm{lo}} \mathrm{CD} 138^{+}\right)$from $\mathrm{Zbp} 1^{\%}(\mathrm{KO}) \mathrm{mice}^{\circ}$ or their wildtype (WT) littermates immunized with just alum (control) or alum-NP-KLH (NP-KLH) on day 10 after immunization. Lack of Zbp1 mRNA confirms Zbp1-deficiency in Zbp 1\% mice. $(\mathrm{n}=3)$. (B, C) Flow-cytometric identification of splenic GCB cells as B220 ${ }^{+} \mathrm{CD} 19^{+} \mathrm{GL} 7^{+} \mathrm{CD} 95^{+}$and GCB frequency in $Z b p 1^{\%}$ mice and their WT littermates immunized with NP-KLH or alum-only control on day 10 after immunization (B) and GCB cell fold-difference between WT and Zbp1\%-mice after NP-KLH immunization normalized to the median frequency of control animals $(C)$. Numbers in the flow cytometry plots represent \% frequency. ( $n=11$ mice/group). (D, E) Flow-cytometric identification of splenic PC as B220 ${ }^{\circ} \mathrm{CD} 138^{+}$and PC frequency in $\mathrm{Zbp1}^{\%}$ mice and their WT littermates in control and NP-KLH-immunized animals on day 10 after immunization (D) and PC fold-difference between WT and Zbp $1^{\%}$ mice after NP-KLH immunization normalized to median frequency of control animals (E). Numbers in the flow cytometry plots represent \% frequency ( $n=11$ mice/group). (F) NP-KLH-specific lgG responses in Zbp $1^{\%}$ mice and their WT littermates in control- and NPKLH-immunized animals on day 10 after immunization. IgG antibody relative levels (left panels) after NP-KLH immunization and their fold-differences normalized to median antibody levels of control animals (right panels). ( $n=6$ mice/group). (G) NP-KLH-specific IgM responses in Zbp $1^{\%}$ mice and their WT littermates in controland NP-KLH-immunized animals on day 10 after immunization. IgM antibody relative levels (left panels) after NP-KLH immunization and their fold-differences normalized to median antibody levels of control animals (right panels) ( $n=6$ mice/group). The error bars of all the cumulative data indicate mean \pm standard error of mean. A two-tailed unpaired $t$-test was applied to determine the $P$ values. ${ }^{*} P \leq 0.05, * * P \leq 0.01, * * * P \leq 0.001, * * * * P \leq 0.0001$, ns- not significant $(P>0.05)$. The number of experiments performed or animals used for the study are indicated separately in each panel legend. 

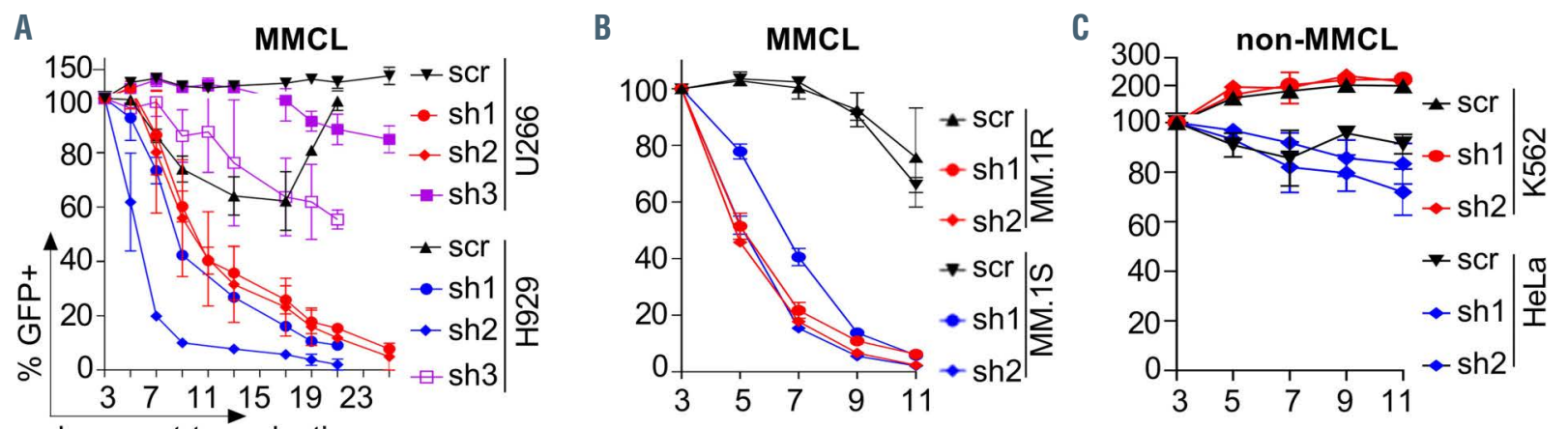

days post-transduction
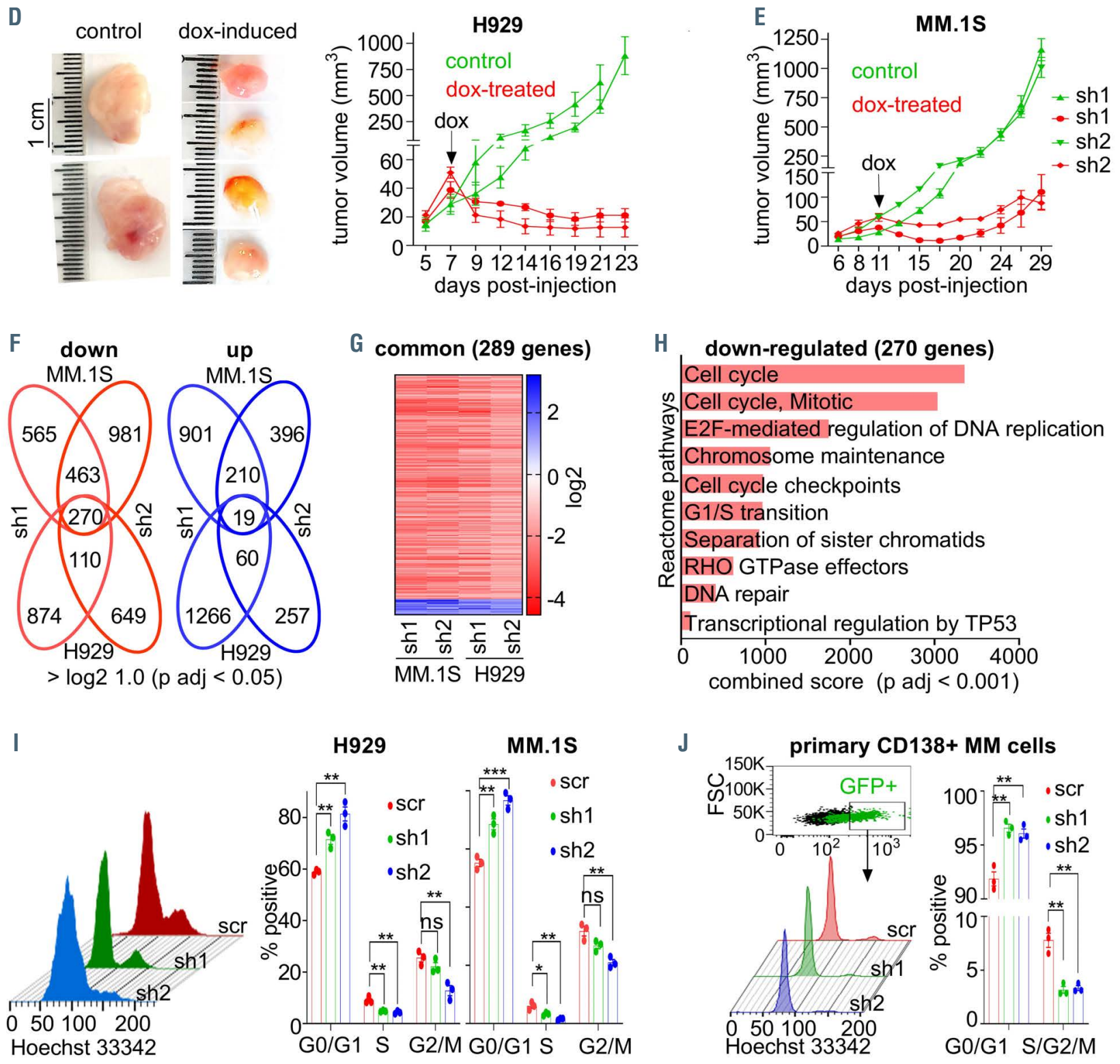

Legend continued on following page

Figure 3. ZBP1 is required for myeloma cell proliferation and survival. (A) Percent green fluorescent protein-positive (\%GFP ${ }^{+}$) cells after transduction with GFP-encoding, ZBP1-targeting shRNA1 (sh1), shRNA2 (sh2), shRNA3 (sh3) or appropriate scrambled control (scr) lentiviral constructs in U266 and H929 cells. All the time points were normalized to day $3 \%$ GFP expression levels for each shRNA or scr control shown. $(n=3)$. (B) \%GFP ${ }^{+}$cells after transduction with ZBP1-targeting sh1, sh2 or appropriate scr control lentiviral constructs in dexamethasone-sensitive MM.1S and its resistant derivative MM.1R cell line. All the time points were normalized to day 3 \%GFP expression levels for each shRNA or scr control shown $(n=3)$. (C) \%GFP cells after the transduction of K562 and HeLa cells, which do not express ZBP1, with ZBP1-targeting sh1 or sh2 or appropriate scr control. The \%GFP ${ }^{+}$cells were normalized to day $3 \%$ GFP expression levels for all the time points for each shRNA or scr control shown $(n=3)$. ( $D, E)$ Photographs of tumors explanted at sacrifice from control, i.e., non-doxycycline (dox)-treated or dox-treated animals engrafted with H929 myeloma cells transduced with dox-inducible sh1 or sh2 targeting ZBP1 (D). Subcutaneous tumor volume of H929 or MM.1S over a period of up to 4 
weeks in control or dox-treated animals (D, E) ( $n=4-5$ mice/group). (F) RNA-sequencing was performed with poly(A) tail-enriched RNA from FACS-purified GFP ${ }^{+}$live cells of scr or shRNA-transduced cells. Venn diagram showing the number of commonly and differentially (up- and down-regulated) expressed genes based on log ${ }_{2}$ fold-change to scr control with a cut-off adjusted $P$-value $(P$ adj $)<0.05$ among the two ZBP1-depleted transcriptomes of the multiple myeloma cell lines (MMCL) H929 and MM.1S and both anti-ZBP1 sh1 or sh2 (n=2). (G) Heatmap showing the expression patterns of the top 289 commonly and differentially expressed genes, based on $\log _{2}$ fold-change to scr control with a cut off Padj <0.05, shared by the two ZBP1-depleted MMCL H929 and MM.1S and both anti-ZBP1 sh1 or sh2. (H) Enrichr pathway enrichment analysis of the shared 270 genes that are commonly downregulated between two MMCL treated with anti-ZBP1 sh1 or sh2 as compared to the scr control. (I) A representative histogram shows depletion of ZBP1 by anti-ZBP1 sh1 or sh2 induces cell cycle arrest in MMCL H929 as compared to the scr control, and its cumulative data shown for MMCL H929 and MM.1S cells. The analysis was performed on GFP ${ }^{+}$cells on day 4 after transduction ( $\mathrm{n}=3$ ). (J) A representative histogram showing that anti-ZBP1 sh1- or sh2-mediated ZBP1 depletion induces cell cycle arrest as compared to the scr control in multiple myeloma (MM) patientderived bone marrow myeloma cells purified for the $\mathrm{CD}_{138^{+}}$marker, and the quantitative data. The flow cytometry plot at the top shows the gating strategy for the $\mathrm{GFP}^{+}$population in transduced cells. The analysis was performed on GFP cells on day 4 after transduction ( $\mathrm{n}=3 \mathrm{MM}$ bone marrow samples). The error bars of all the cumulative data indicate the mean \pm standard error of mean. A two-tailed unpaired $t$-test was applied to determine the $P$ values. $* P \leq 0.05$, $* \star P \leq 0.01$, $* * * P \leq 0.001, * * * * P \leq 0.0001$, ns: not significant $(P>0.05)$. The number of experiments performed is indicated separately in each panel legend.

the role of ZBP1 in GCB and PC development, we assessed the humoral immune responses against the T-cell-dependent antigen NP-KLH using alum as an adjuvant. We found that while Zbp1 mRNA is undetectable in FACS-purified GCB cells and PC from $Z b p 1^{-1-}$ mice in response to either NP-KLH-alum or alum-only control, it increased from GCB cells to PC in wild-type (WT) littermates and this increase was more pronounced upon immunization with NP-KLH-alum (Figure 2A).

Splenic frequencies of $\mathrm{B} 220^{+} \mathrm{CD} 19^{+} \mathrm{GL} 7^{+} \mathrm{CD} 95^{+} \mathrm{GCB}$ cells (Figure $2 \mathrm{~B}, \mathrm{C}$ ) and $\mathrm{B} 220^{\mathrm{lo}} \mathrm{CD} 138^{+} \mathrm{PC}$ (Figure 2D, E) were not different at baseline between $Z b p^{1-1}$ and their WT littermates. Similarly GCB-cell frequencies were not significantly different between WT and $Z b p 1^{-1-}$ animals after NP-KLH-alum immunization; however, the increase in PC frequency and NP-KLH-specific IgG (but not IgM) serum levels in immunized $Z b p 1^{-1}$ animals was significantly lower compared to that in immunized WT littermates (Figure 2D-G). These findings suggest that although Zbp1 is not required for GCB cell and PC development under steady-state, it is required for optimal T-cell-dependent humoral immune responses. Whether cellular nucleic acids in complex with Zbp1 play a role in this process remains to be addressed.

\section{ZBP1 is required for myeloma cell proliferation and survival}

To investigate the functional role of ZBP1 in MM, we depleted ZBP1 expression in MMCL by targeting both of its main isoforms, i.e., isoform 1 comprising $Z \alpha 1$ and $Z \alpha 2$ domains and isoform 2 which lacks $Z \alpha 1$. Depletion of either isoform 1 or both isoforms 1 and 2 by shRNA1- or shRNA2-mediated knockdown, respectively, was toxic to H929 and U266 cells while shRNA3 did not deplete ZBP1 and behaved like the scrambled control without affecting cell viability (Figure $3 \mathrm{~A}$ and Online Supplementary Figure $S 3 A-C)$. Depletion of ZBP1 by shRNA1/2 was also toxic to MMCL MM.1S and its dexamethasone-resistant derivative MM.1R (Figure $3 \mathrm{~B}$ and Online Supplementary Figure $S 3 D)$. These findings suggest that the observed effect is mediated by depletion of isoform 1 and cannot be rescued by isoform 2. This effect was specific because the antiproliferative function was not observed in the shRNAtransduced erythromyeloid K562 or epithelial HeLa cells (Figure 3C) which lack ZBP1 expression (Online Supplementary Figures $S 1 F$ and $3 E$ ). Furthermure, depletion of shRNA1-transduced myeloma cells was at least in part rescued by overexpression of $Z B P 1 \mathrm{cDNA}$ with appropriate silent mutations (Online Supplementary Figure $S 3 F$ ). Mutating the seed region of shRNA1, aimed at eliminating off-target effects, ${ }^{35}$ did not alter either the expression of ZBP1 or the cytotoxic effects in MM.1S cells (Online Supplementary Figure $S 3 G, H)$. Using a doxycycline- inducible shRNA, ${ }^{36}$ we found that ZBP1 depletion inhibited myeloma cell growth in vitro (Online Supplementary Figure $S 3 I, J)$ and also subcutaneous myeloma tumor growth in vivo (Figure 3D, E and Online Supplementary Figure $S 3 K, L)$. Together, these findings suggest an important role of ZBP1 in myeloma cell biology.

In line with these observations, transcriptome analysis of two ZBP1-depleted MMCL, in which oncogenic transcriptomes are driven by MAF (MM.1S) or MMSET (H929) oncogenes, revealed 270 genes that are significantly downregulated in both cells and by both shRNA (Figure 3F, G and Online Supplementary Table S1). These genes were highly enriched for cell cycle control pathways (Figure 3H and Online Supplementary Table S2).

We also validated reduction of the mRNA expression levels of the cell cycle regulators Ki-67, FOXM1 and E2F1 upon ZBP1-depletion by quantitative polymerase chain reaction analysis (Online Supplementary Figure $S 4 A, B$ ) and confirmed the decrease in proteins by immunoblotting (FOXM1 and E2F1) and flow-cytometry (Ki-67) (Online Supplementary Figure S4C-E). Flow-cytometric analysis of the cell cycle in ZBP1-depleted cells revealed arrest at the G0/G1 phase (Figure 3I) in conjunction with increased apoptosis as assessed by annexin V staining in MM.1S and H929 cells (Online Supplementary Figure S4F). Notably, we also confirmed that both anti-ZBP1 shRNA induced cell cycle arrest in $\mathrm{MM}$ patient-derived, bone marrow myeloma CD $138^{+}$PC (Figure 3J and Online Supplementary Figure $S 4 G)$, thus confirming the role of ZBP1 in cell cycle regulation in primary myeloma PC as well as MMCL.

In addition to downregulation of cell cycle pathways, GSEA also showed significant enrichment for the IFN type I pathway in upregulated genes induced by both shRNA1 and shRNA2 in MM.1S cells but in downregulated genes by only shRNA1 in H929 cells (Online Supplementary Figure $54 H$ ). This disparate effect of ZBP1 depletion on IFN type I response genes might reflect the distinct transcriptomes of MM.1S and H929 MMCL imposed by their primary driver oncogenes. Previous work demonstrated that a transcriptional proliferative signature identifies a minority of $\mathrm{MM}$ patients with adverse prognosis. ${ }^{37,38}$ Accordingly, GSEA of myeloma PC transcriptomes with the top $5 \%$ highest versus $90 \%$ lowest ZBP1 expression revealed significant enrichment in the former for cell cycle regulation pathways among overexpressed genes (Online Supplementary Figure S4I, J and Online Supplementary Table S3). Interestingly, among these overexpressed genes in the subgroup of $\mathrm{ZBP} 1^{\text {hi }}$ patients, we also observed significant enrichment for IFN type I signaling consistent with the role of ZBP1 as an IFNresponse gene (Online Supplementary Figure S4I, J and Online Supplementary Table S3). 


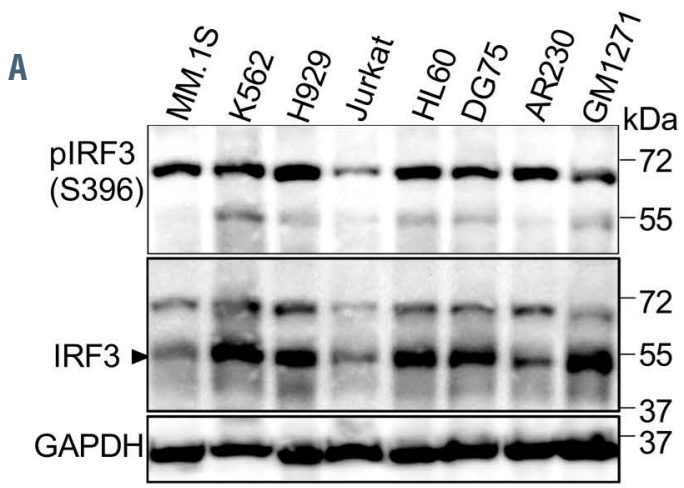

C
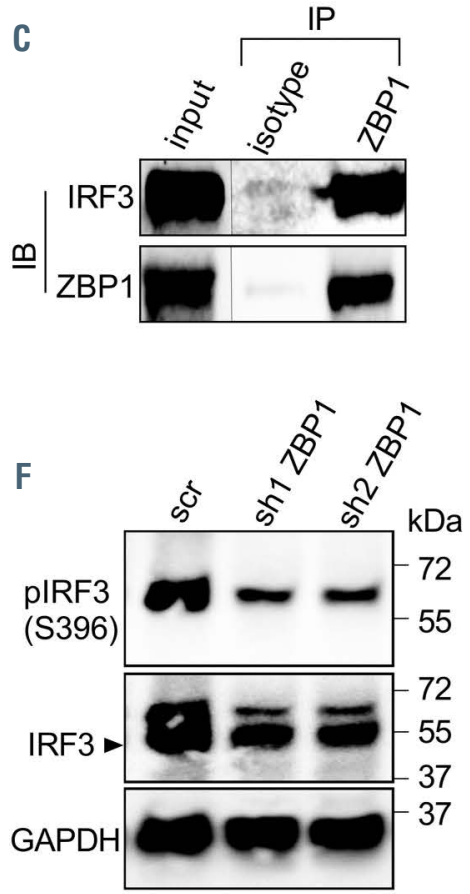

shIRF3

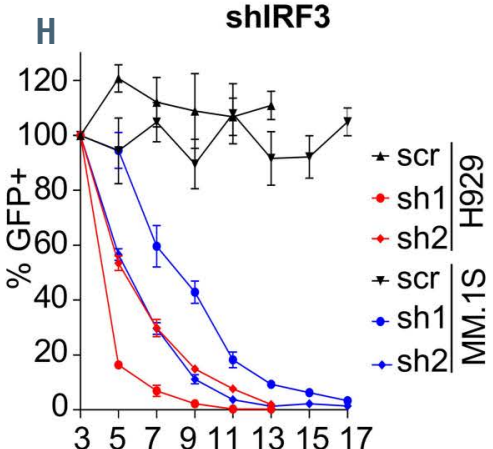

357911131517

days post-transduction

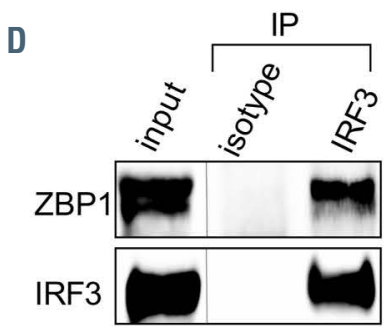

B
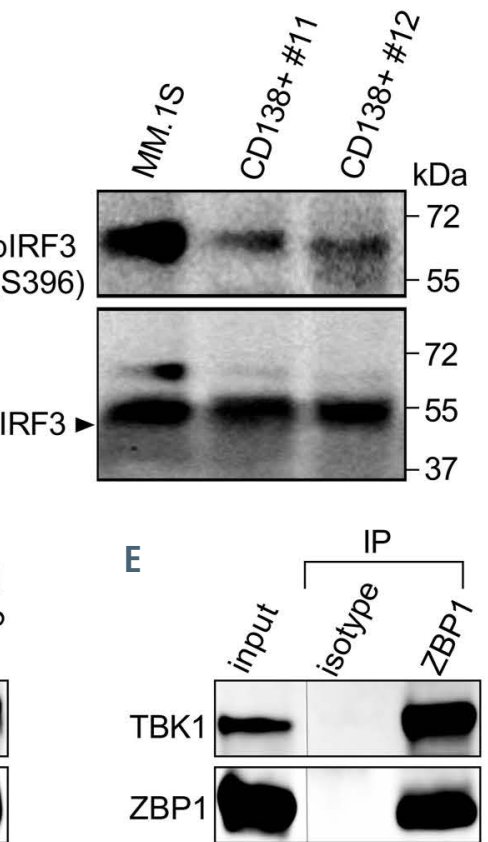

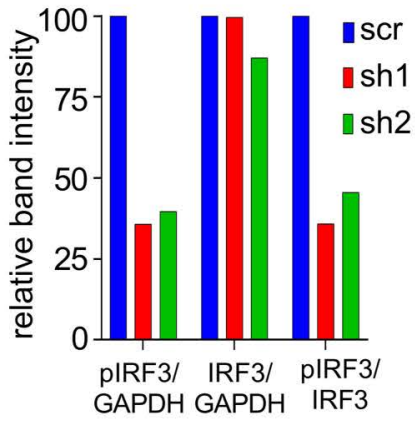

I

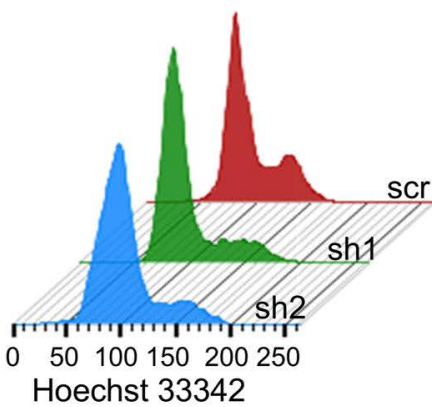

G

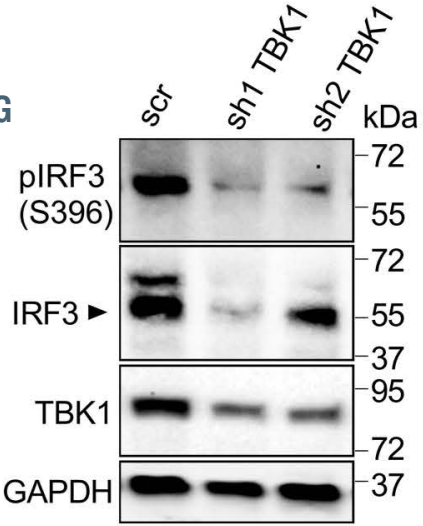

H929

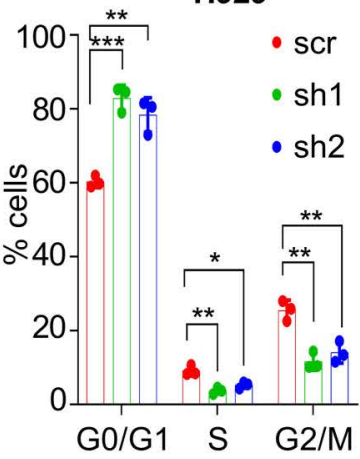

Figure 4. ZBP1 as a scaffold for IRF3 constitutive activation by TBK1. (A) Immunoblotting analysis of pIRF3/IRF3 expression in myeloma (MM.1S and H929) and non-myeloma cell lines (K562; Jurkat; HL60: acute myeloid leukemia; DG75: B lineage; AR230: chronic myeloid leukemia; GM1271: Epstein-Barr virus-transformed B-cell lineage). (B) Immunoblotting for pIRF3/IRF3 expression in MM patient-derived bone marrow plasma cells (PC) purified for CD138 ${ }^{+}$using $\mathrm{CD} 138$ immunomagnetic microbeads. (C, D) Immunoblotting against IRF3 and ZBP1 in MM.1S cells following co-immunoprecipitation with anti-ZBP1 (C), or anti-IRF3 (D) or corresponding isotype control antibodies. (E) Immunoblotting against TBK1 and ZBP1 in MM.1S cells following co-immunoprecipitation with anti-ZBP1 or its corresponding isotype control antibodies. (F) Immunoblotting for PIRF3/IRF3 expression in anti-ZBP1 shRNA1 (sh1) or shRNA2 (sh2) or scrambled (scr) control RNA transduced MM1S cells on day 4 after transduction (left). ImageJ quantification shows profound reductions in pIRF3 but not in total IRF3 levels in anti-ZBP1 sh1- or sh2-transduced cells cells asconpared to scr control cells (right). The expression on day 4 following anti-TBK1 sh1, sh2 or scr transduction in MM.1S cells. The protein lysates were prepared from the cells with >90\% transduction efficiency. (H) Percentage green fluorescent protein-positive (\%GFP) cells after transduction with IRF3-targeting sh1, sh2 or scr control in MMCL H929 and MM.1S cells. All the time points were normalized to day $3 \%$ GFP expression levels for each shRNA shown $(n=3)$. (I) A representative flow-cytometric histogram of cell cycle in MMCL transduced with anti-IRF3 sh1 or sh2 or scr control. Analysis was performed on GFP ${ }^{+}$cells day 4 after transduction and the cumulative data for H929 show cell cycle arrest in anti-IRF3 sh1- or sh2-transduced H929 cells $(n=3)$. The error bars of all the cumulative data indicate mean \pm standard error of mean. A two-tailed unpaired t-test was applied to determine the $P$ values. $* P \leq 0.05, * * P \leq 0.01, * * * P \leq 0.001$. The number of experiments performed for the study is indicated separately in each panel legend. 


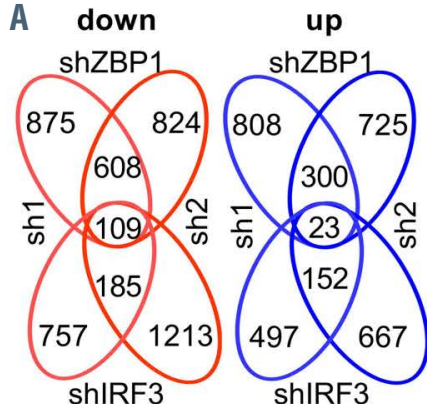

top $50 \% \log 2(p$ adj $<0.05)$
B top $50 \%$ (132 genes)

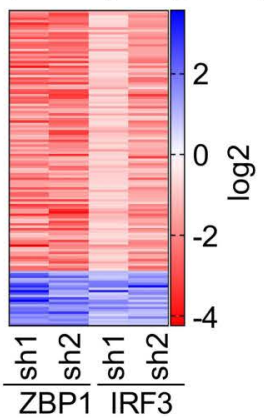

C down-regulated (109 genes) G1/S-specific transcription (2) E2F-mediated regulation of DNA replication 3 Cell cycle TP53 regulates transcription of cell cycle genes ¿ Cell cycle checkpoints है G2/M checkpoints HDR through homologous recombination (HRR) RHO GTPases activate formins DNA repair

Separation of sister chromatids

$\begin{array}{lllll}0 & 250 & 500 & 750 & 1000\end{array}$ combined score ( $p$ adj < 0.05)

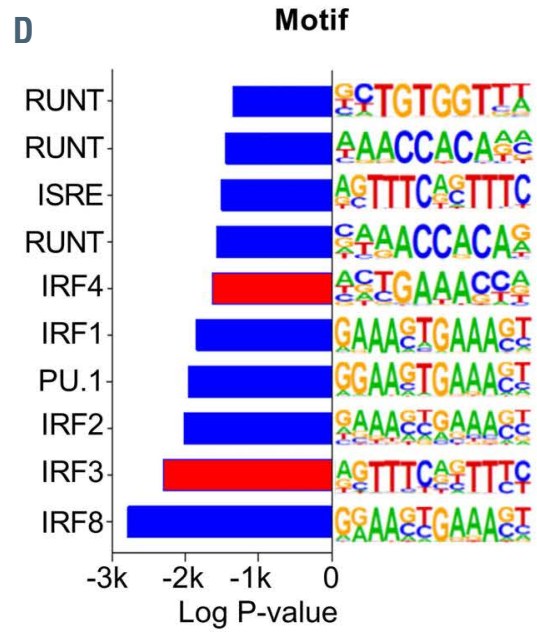

$\mathbf{F}$

Activated

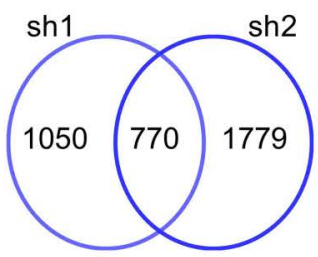

Repressed

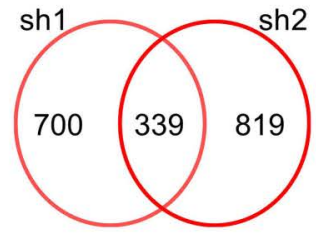

G

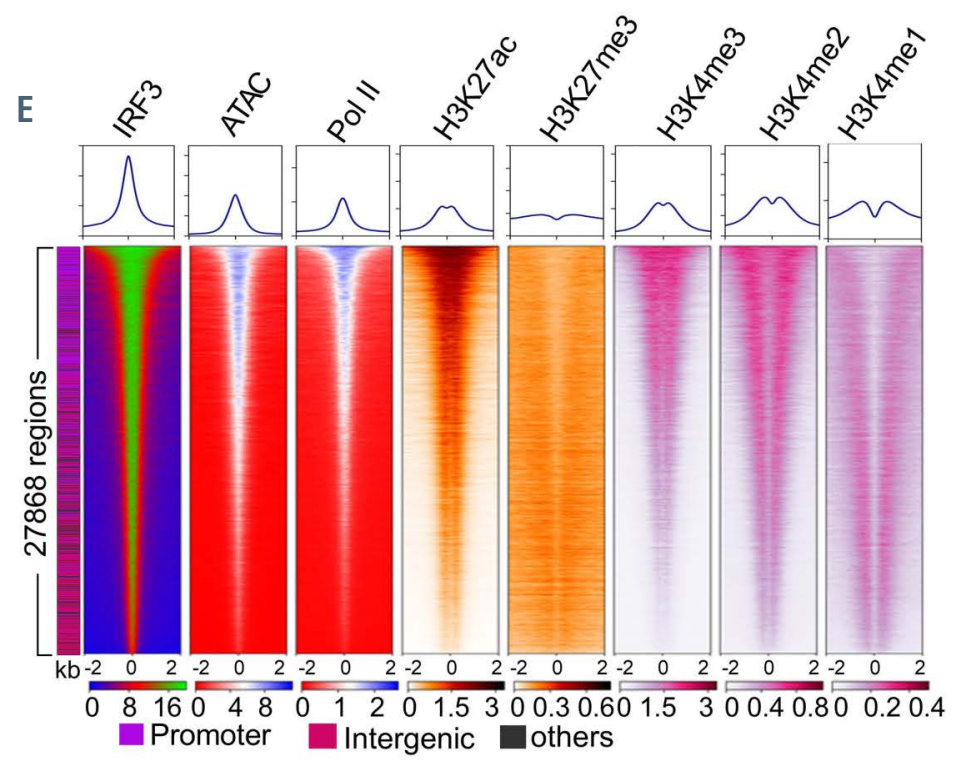

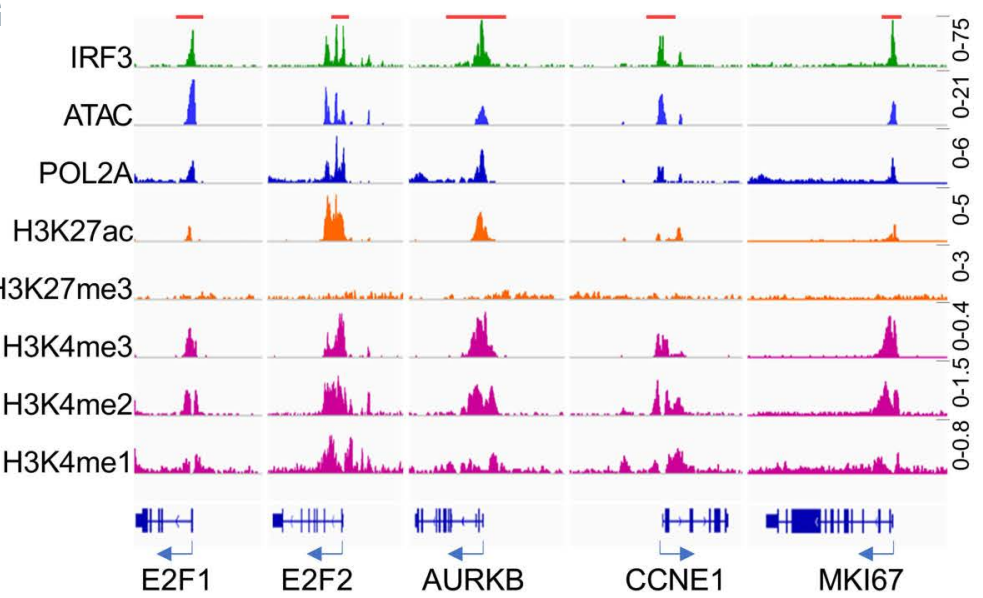

Figure 5. IRF3 regulates cell cycle genes in myeloma cells. (A) RNA-sequencing was performed with poly(A) tail-enriched RNA from FACS-purified green fluorescent protein (GFP) ${ }^{+}$live cells of scrambled (scr) or shRNA-transduced cells. The Venn diagram shows the numbers of commonly up- and down-regulated genes among the top 50\% differentially expressed genes, based on $\log _{2}$ fold-change to the scr control with cut-off Padj <0.05, of ZBP1-depleted and IRF3-depleted transcriptomes in MM.1S cells $(n=2)$. (B) Heatmap showing the expression patterns of the top 132 commonly expressed genes among the top $50 \%$ differentially expressed genes of ZBP1-depleted and IRF3-depleted transcriptomes in MM.1S cells. (C) Enrichr pathway enrichment analysis for the shared 109 genes that are downregulated in common upon ZBP1- or IRF3-depletion in MM.1S cells. (D) Transcription factor motif analysis of IRF3 chromatin immunoprecipitation (ChIP)-sequencing shows enrichment for IRF3-bound genomic regions in MM.1S cells (IRF3 ChIP-sequencing; $n=2$ ). (E) Metagene (top) and heatmap (bottom) representation of IRF3 genome-wide binding as assessed by IRF3 ChIP-sequencing in MM.1S cells along with indicated histone marks, RNA polymerase II (Pol II) binding and chromatin accessibility as assessed by ATAC-sequencing. Genomic feature annotation for each peak in the heatmap is shown on the left and the small color bar (below) indicates the genomic regions for 27,868 binding regions annotated. IRF3 binding is observed in genomic regions marked for active transcription i.e., with increased chromatin accessibility, activating chromatin marks (H3K27ac, H3K4me1/3) and Pol II binding. (F) Venn diagram showing numbers of genes predicted to be directly regulated (activated or repressed) by IRF3 in MM.1S cells as assessed by integration of IRF3 cistrome, IRF3 binding within 2 kb distance of the transcription start site, and IRF3-depleted transcriptome with cut-off Padj $<0.05$ using BETA-plus software. (G) IGV browser snapshots of IRF3 and Pol II binding, chromatin accessibility and histone mark enrichment at regulatory areas of several genes promoting cell cycle progression and cell proliferation. The red block on the top indicates $5 \mathrm{~kb}$ genome size. 
A

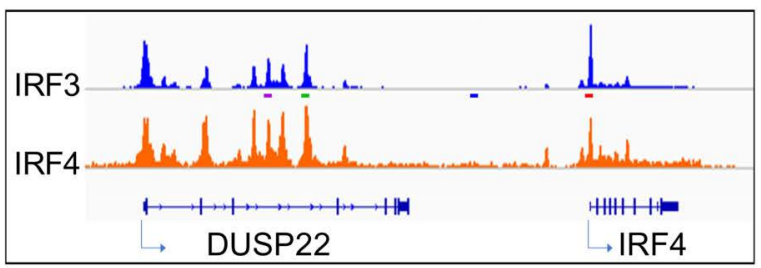

B

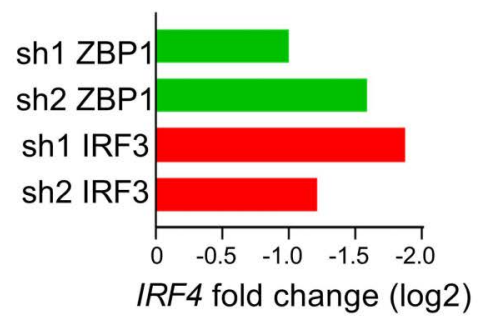

F $\quad$ Activated (612 genes)

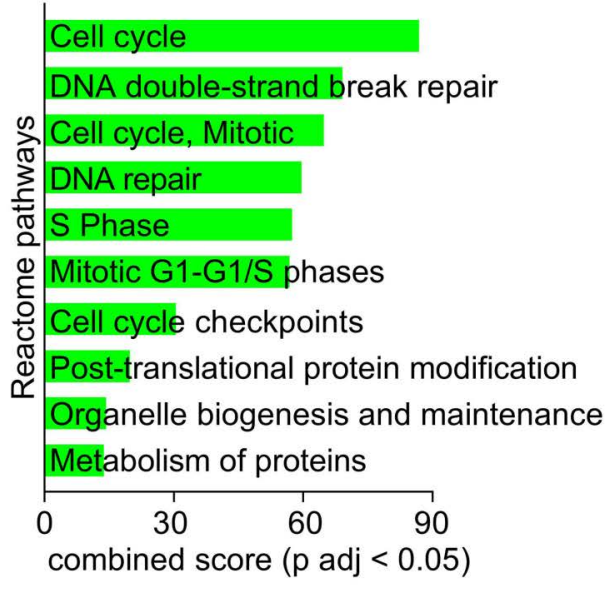

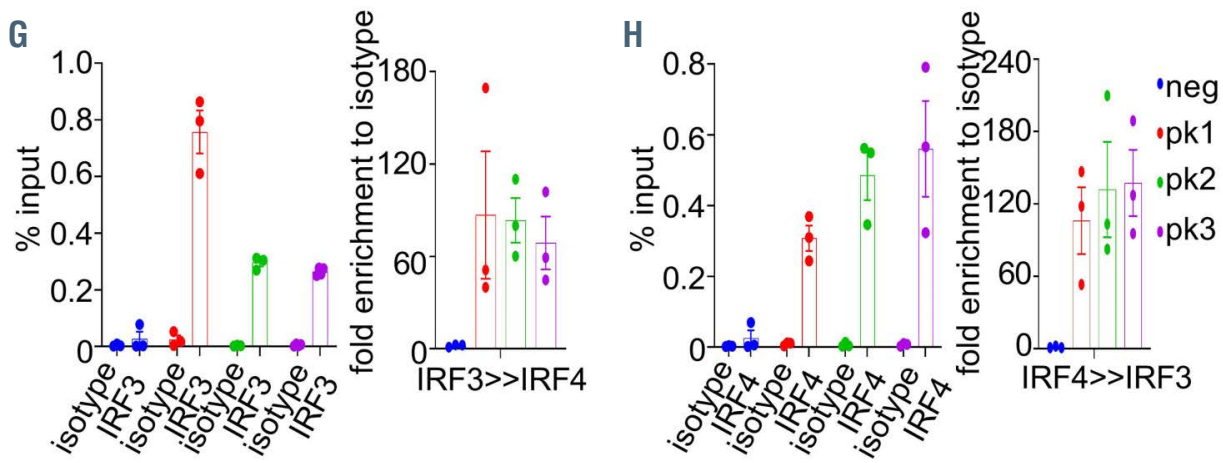

Figure 6. IRF3 co-operates with IRF4 and regulates cell cycle genes in myeloma cells. (A, B) IGV browser snapshots of IRF3 and IRF4 co-binding at the promoter and super-enhancer of IRF4 as assessed by chromatin immunoprecipitation (ChIP)-sequencing (A) and log fold-change of IRF4 expression (Padj $<0.05$ ) assessed by RNA-sequencing after depletion of indicated mRNA/protein in relation to the scrambled (scr)control in MM.1S cells (B). (C) Heatmaps of IRF3 and IRF4 genome-wide binding, and common binding regions of IRF3 and IRF4 (intersection) as their binding regions are intersected by Bedtools Intersect. ${ }^{50}$ Numbers of binding regions are shown in brackets. (D) Venn diagram showing the numbers of genomic regions of IRF3 and IRF4 co-binding with gene regulatory potential as assessed by integration of the whole transcriptome of IRF3-depleted MM.1S cells with IRF3- or IRF4-cistrome alone or IRF3 and IRF4 co-binding regions (intersection) in MM.1S cells. Here IRF4 genome-wide binding regions with very low scores were omitted and only the top $50 \%$ binding regions with highest scores were used. (E) Numbers of genes predicted to be directly co-regulated (repressed or activated) by IRF3 and IRF4 binding. The co-binding (intersection) regions were integrated with the IRF3-depleted transcriptome by shRNA1 and shRNA2 in MM.1S cells. (F) Enrichr pathway enrichment analysis for common genes predicted to be activated by IRF3-IRF4 co-binding. (G) Primary ChIP quantitative polymerase chain reaction (qPCR) against IRF3 (left) followed by re-ChIP-qPCR against IRF4 (right). The position of amplicons is shown as horizontal colored lines in Figure 6A. (H) Primary ChIP-qPCR against IRF4 (left) followed by re-ChIP-qPCR against IRF3 (right). The position of amplicons is shown as horizontal colored lines in Figure 6A.

\section{ZBP1 interaction with IRF3 and TBK1}

We next investigated the downstream processes that might link constitutive ZBP1 expression in myeloma cells with regulation of cell cycle. Unlike in non-malignant cells in which IRF3 phosphorylation/activation requires activation of sensors such as CGAS-STING, ${ }^{14,16}$ we found that IRF3 was constitutively phosphorylated (pIRF3) in myeloma cell lines (Figure 4A). In line with previous reports,39 pIRF3 was also detected in other non-myeloma cancer cells (Figure 4A). Importantly, pIRF3 was also detected in primary $\mathrm{BM}$ myeloma $\mathrm{CD} 138^{+} \mathrm{PC}$ (Figure $4 \mathrm{~B}$ ) and thus establishing that IRF3 is constitutively phosphorylated in MM. Although the functional relationship of the ZBP1-IRF3 interaction in the context of cellular innate immune responses is a matter of debate, the physical interaction of ZBP1-IRF3 was previously demonstrated by their ectopic expression. ${ }^{17,40,40}$ Using protein co-immunoprecipitation assays we found that endogenous ZBP1 interacts with endogenous IRF3 (Figure 4C, D) and TBK1 (Figure 4E) in MM.1S cells. By co-transfection of IRF3 cDNA with a full length or C-terminus deleted mutant of ZBP1 (Online Supplementary Figure S5A), we found that the 
ZBP1-IRF3 interaction requires the RHIM domain-containing C-terminus of ZBP1 (Online Supplementary Figure $S 5 B, C)$. Furthermore, while total IRF3 and TBK1 levels were not appreciably altered in ZBP1-depleted cells, pIRF3 and $\mathrm{pTBK} 1$ levels markedly decreased in both constitutive shRNA-transduced (Figure 4F) and doxycycline-induced shRNA targeting ZBP1 (Online Supplementary Figure S5D) in MM.1S cells. These findings suggest a post-translational dependency of IRF3 and TBK1 constitutive phosphorylation on ZBP1. Finally, shRNA-mediated depletion of TBK1 resulted in a decrease of IRF3 phosphorylation (Figure 4G). Together these data support a model whereby ZBP1 serves as a scaffold for TBK1-dependent constitutive phosphorylation of IRF3 in MMCL.

\section{IRF3 regulates the cell cycle in myeloma cells}

As observed for ZBP1, IRF3 depletion also induces cell cycle arrest and apoptosis and thereby inhibits myeloma cell growth (Figure 4H, I and Online Supplementary Figure $S 6 A-C)$. Of note, a similar effect was observed after depletion of TBK1 (Online Supplementary Figure S6D-G). In addition, transcriptome analysis of IRF3-depleted MM.1S cells revealed that among 185 genes downregulated by both anti-IRF3 shRNA1 and shRNA2, 109 genes were also commonly downregulated upon ZBP1 depletion (Figure 5A, B and Online Supplementary Table S4) and these are also enriched for cell cycle regulation (Figure 5C and Online Supplementary Table S5). However, only 23 genes were shared among those upregulated upon depletion of ZBP1 or IRF3.

To identify candidate transcriptional targets of IRF3 in myeloma cells we generated and mapped its genomewide binding by IRF3 ChIP-sequencing in MM.1S cells. IRF3-bound regions (promoter, intergenic and intronic) (Online Supplementary Figure S7A) were highly enriched for IRF3-binding motifs (Figure 5D). In the same cells, we correlated genome-wide IRF3 binding with chromatin accessibility as assessed by an assay for transposase-accessible chromatin (ATAC)-sequencing, RNA polymerase II binding, and activating (H3K27ac and H3K4me1/2/3) and repressive (H3K27me3) histone marks (Figure $5 \mathrm{E}$ ). This showed that IRF3 binding occurs in nearly 28,000 highly accessible chromatin regions with activating transcriptional potential as revealed by Pol II binding and the presence of activating histone marks. Thus, constitutively phosphorylated IRF3 in myeloma cells is highly transcriptionally active in the nucleus.

Next, to obtain the compendium of genes directly regulated by IRF3 in MM.1S cells, we integrated the IRF3depleted transcriptome for each anti-IRF3 shRNA with the IRF3 cistrome using BETA-plus software (Figure 5F and Online Supplementary Figure S7D). After intersection of anti-IRF3 shRNA1 and shRNA2 data, we found that the 770 genes predicted to be directly activated by IRF3, included the key cell cycle regulators E2F1, E2F2, AURKB, CCNE1, MKI67 and MCM2-7 complex (Figure 5G, Online Supplementary Figure S7E, F and Online Supplementary Table $S 6)$, and were significantly enriched for cell cycle regulation pathways (Online Supplementary Figure S7B and Online Supplementary Table S7). Notably, the 339 genes predicted to be repressed by IRF3 were not enriched for IFN type I response genes (Figure 5F, Online Supplementary Figure S7C and Online Supplementary Tables S6 and S7). Consistent with this, while IRF3 binds in the regulatory regions of IFNA1 (but not of IFNB1) and ISG15, which are hallmark type I IFN response genes, expression of these genes was not altered upon IRF3 depletion (Online Supplementary Figure $S 7 G, H)$.

IRF3 regulates transcription of and co-operates with IRF4 to regulate the cell cycle in myeloma cells. IRF4 is a transcription factor critical for normal PC development ${ }^{34}$ while in myeloma PC it co-operates with MYC to establish a transcriptional circuitry to which myeloma cells are highly addicted. ${ }^{41}$ Since the IRF4 motif was among the top-most enriched regions bound by IRF3 (Figure 5D), we explored potential synergy between IRF3 and IRF4 by overlaying our in-house-generated genome-wide binding profile of IRF3 with that of previously published IRF4 ChIP-sequencing in MM.1S cells. ${ }^{42}$ First, we observed cobinding of the two transcription factors at the promoter and the previously established super-enhancer of IRF $4^{43}$ (Figure 6A). This observation and the fact that IRF4 expression is significantly downregulated following IRF3 as well as ZBP1 depletion (Figure 6B) suggested that IRF4 transcriptional regulation is, at least in part, under control of the ZBP1-IRF3 axis.

At a genome-wide level, 21,614 IRF3-bound chromatin regions were co-bound by IRF4 (Figure 6C, D). Correlating these with transcriptome changes following IRF3 depletion, we identified 612 and 267 genes predicted to be coactivated or co-repressed, respectively, by both IRF3 and IRF4 (Figure 6D, E) with the former highly enriched in cell cycle regulators (Figure 6F and Online Supplementary Tables S8 and S9). To validate this on-chromatin association of IRF3 with IRF4, we performed ChIP-re-ChIP assays at IRF3-IRF4 co-binding regions using a region upstream of IRF4 in which no binding of either transcription factor was observed as a negative control. In all tested regions we found specific co-occupancy of IRF3 and IRF4 including in the IRF4 promoter and super-enhancer regions (Figure 6G, $\mathrm{H})$ and also at genes regulating the cell cycle including E2F1, E2F2, MCM2 and AURKB (Online Supplementary Figure $S 8 A, B)$.

\section{Discussion}

Here we demonstrate that the Z-nucleic acid sensor ZBP1 is an important and novel determinant of MM biology. We link the myeloma-selective constitutive expression of ZBP1 to constitutive IRF3 activation and regulation of IRF3-dependent IRF4 expression and myeloma cell proliferation.

While other nucleic acid sensors, e.g., cGAS-STING, are expressed constitutively and are activated upon nucleic acid binding, ${ }^{14,16} \mathrm{ZBP} 1$ differs in that its expression is only detected in response to nucleic acids, viral pathogens or inflammatory stimuli including interferons. . $7,8,20,44^{\text {Our }}$ extensive analysis confirmed that ZBP1 expression is low or not detected in all human normal and cancer cells tested with the striking exception of cells in the late B-cell development trajectory and in particular PC. Reflecting their cell of origin, we found constitutive ZBP1 expression also in MMCL and primary myeloma PC.

While our data do not address the molecular role of Zbp1 in late B-cell development, at a cellular level, they identify suboptimal humoral immune response to a T-celldependent antigen in $Z b p 1^{-/-}$mice. Of note, in contrast to our results, antibody levels in $Z b p^{1-1-}$ mice were previously found to be intact in response to DNA vaccination; ${ }^{18}$ the 
different routes and process of immunization might account for these differences.

We found that ZBP1 depletion had a profound and selective effect on MMCL proliferation and survival in vitro and in vivo. Similarly, in primary myeloma PC which are less proliferative than MMCL, depletion of ZBP1 also induced cell cycle arrest. Based on appropriate design of $Z B P 1$-targeting shRNA we could determine that myeloma cell proliferation is sustained by the isoform 1 , which retains both $Z \alpha 1$ and Z $\alpha 2$ domains, but not by the isoform 2, which retains only the $Z \alpha 2$ domain. Future research will explore the nature and origin of nucleic acids that are bound by the $Z \alpha$ domain and their impact on the pro-proliferative function of ZBP1 in myeloma cells.

Transcriptomes of ZBP1-depleted myeloma cells, which are driven by distinct primary oncogenes, i.e., $M A F$ (MM.1S cells) and MMSET (H929 cells), highlighted cell cycle regulation as one of the main pathways regulated by $\mathrm{ZBP} 1$. This novel pro-proliferative function of ZBP1 contrasts with the anti-proliferative potential of IFN $\beta$ which can induce and sustain expression of ZBP1. ${ }^{45,20}$ However, although GSEA suggested that ZBP1 mediates repression of IFN type I response in ZBP1-depleted MM.1S cells, study of a large number of primary myeloma PC transcriptomes revealed a strong IFN type I response transcriptional signature as well as enrichment for cell cycle pathways among upregulated genes in ZBP1 ${ }^{\text {hi }}$ myeloma PC. Together, these findings are consistent with a model whereby an active IFN type I response restrains myeloma PC proliferation in the low proliferative early phase myeloma PC but sustains ZBP1 expression, which exerts a limited pro-proliferative function. In contrast, while the IFN type I transcriptional program is attenuated or even repressed in the highly proliferative MMCL such as MM.1S cells, which are representative of advanced $\mathrm{MM},{ }^{21,46}$ persistent $\mathrm{ZBP} 1$ expression regulates proliferation which is not constrained by the IFN type I response. In line with this model, a recent comparison of primary myeloma $\mathrm{PC}$ and MMCL transcriptomes demonstrated enrichment for the IFN type I response gene signature in myeloma PC in more than $700 \mathrm{MM}$ patients at diagnosis while proliferative but not IFN type I gene signatures were dominant in relapsed disease myeloma PC and in MMCL. ${ }^{21}$

The physiological role of ZBP1 is to promote necroptosis and inflammation through interaction with RIPK3 in response to pathogens or cellular dsRNA. ${ }^{47,10,12}$ While interaction of ZBP1 with IRF3 and TBK1 has been shown previously in an ectopic expression system, ${ }^{17,40}$ whether it regulates the type I IFN response has been disputed. ${ }^{18} \mathrm{Here}$ we confirmed direct and functional interactions of endogenous ZBP1-IRF3-TBK1 in myeloma cells which highlights ZBP1 as a physical platform that directs activation of TBK1 and IRF3.

While transcriptional activation by phosphorylation of IRF3 in response to inflammatory stimuli is expected to be transient, we found that IRF3 is constitutively phosphorylated in both primary myeloma cells and cell lines. This is not unique to $\mathrm{MM}$ since constitutively phosphorylated IRF3 has been reported in several ZBP1-negative cancer lines $^{39}$ but not functionally investigated although a proproliferative effect of IRF3 has been reported in acute myeloid leukemia cells at a cellular level. ${ }^{48}$ Importantly, we demonstrate that IRF3 binds to transcriptionally active regions of the genome and it directly regulates genes that promote cell cycle progression in myeloma cells. Accordingly, IRF3 depletion in myeloma cells leads to cell cycle arrest and apoptosis.

We also show that TBK1 depletion results in cell cycle arrest and apoptosis in myeloma cells. Although this cellular effect is likely linked to downstream regulation of cell cycle genes by pIRF3, other mechanisms are also possible since TBK1 is a pleiotropic kinase. ${ }^{49}$

IRF4, the lineage-defining transcription factor in PC development, ${ }^{34}$ establishes an aberrant transcriptional circuity with MYC that renders myeloma PC highly dependent on an oncogenic program that includes activation of the cell cycle among other pathways. ${ }^{41}$ Here, based on IRF3 binding to the super-enhancer and promoter of IRF4 and the fact that depletion of IRF3 (also ZBP1) results in significant IRF4 downregulation, we demonstrate direct transcriptional activation of IRF4 by IRF3. Indeed, since as little as $50 \%$ reduction in IRF4 expression levels is toxic to myeloma cells, ${ }^{41}$ the greater than $50 \%$ reduction in IRF 4 mRNA induced by IRF3 depletion would be expected to contribute significantly to myeloma cell death. Our genome-wide and sequential ChIP assays demonstrated and validated extensive co-occupancy of IRF3 and IRF4 in the myeloma regulatory genome including at the superenhancer of IRF4, with genes involved in cell cycle control being among the targets of the IRF3-IRF4 synergy.

In summary, our data show that like other nucleic acid sensors, ZBP1 can regulate cellular pathways critical for cancer biology. We show a constitutively active ZBP1-IRF3 axis that is co-opted into promoting proliferative pathways in myeloma PC and regulating expression of the critical myeloma oncogene IRF4. Guided by our initial delineation of the structural requirements of the ZBP1-IRF3 interaction in myeloma cells, disruption of the ZBP1-IRF3 axis will offer an opportunity for targeted and relatively selective therapeutic intervention in MM.

\section{Disclosures}

No conflicts of interest to disclose.

\section{Contributions}

$K P$ and $A K$ conceived and designed the study; KP and MMT performed the co-immunoprecipitation and doxycycline-inducible study in vitro; KP and MB performed the shTBK1 study. PT and $K N$ performed immunohistochemistry; $A C$ processed RNAsequencing data for ZBP1; MER processed RNA-sequencing and ChIP-sequencing data for ZBP1 and IRF3; KP performed all other experiments in vitro, in vivo and integrated all the ChIP-sequencing and RNA-sequencing data and performed all other bioinformatics analysis and created all the figures; DI provided erythroblast cells; VSC, AK, NT, XX, IVK, IR, and HWA provided reagents; AK supervised the study. $K P$ and $A K$ wrote the manuscript.

\section{Funding}

We acknowledge funding from Blood Cancer UK (to KP, NT, $X X$, and VC), KKLF (to AK), and the Imperial NIHR Biomedical Research Centre, LMS/NIHR Imperial Biomedical Research Centre Flow Cytometry Facility, Imperial BRC Genomics Facility and the MRC/LMS Sequencing Facility for support. 


\section{References}

1. Palumbo A, Anderson K. Multiple myeloma. N Engl J Med. 2011;364(11):1046-1060.

2. Morgan GJ, Walker BA, Davies FE. The genetic architecture of multiple myeloma. Nat Rev Cancer. 2012;12(5):335-348.

3. Manier S, Salem KZ, Park J, Landau DA, Getz G, Ghobrial IM. Genomic complexity of multiple myeloma and its clinical implications. Nat Rev Clin Oncol. 2017; 14(2):100-113.

4. Shi W, Liao Y, Willis SN, et al. Transcriptional profiling of mouse B cell terminal differentiation defines a signature for antibody-secreting plasma cells. Nat Immunol. 2015;16(6):663-673.

5. Ha SC, Kim D, Hwang H-Y, Rich A, Kim Y-G, Kim KK. The crystal structure of the second Z-DNA binding domain of human DAI (ZBP1) in complex with Z-DNA reveals an unusual binding mode to ZDNA. Proc Natl Acad Sci U S A. 2008;105 (52):20671-20676.

6. Schwartz T, Behlke J, Lowenhaupt K, Heinemann U, Rich A. Structure of the DLM-1-Z-DNA complex reveals a conserved family of Z-DNA-binding proteins. Nat Struct Biol. 2001;8(9):761-765.

7. Maelfait J, Liverpool L, Bridgeman A, Ragan KB, Upton JW, Rehwinkel J. Sensing of viral and endogenous RNA by ZBP1/DAI induces necroptosis. EMBO J. 2017; 36(17):2529-2543.

8. Thapa RJ, Ingram JP, Ragan KB, et al. DAI senses influenza A virus genomic RNA and activates RIPK3-dependent cell death. Cell Host Microbe. 2016;20(5):674-681.

9. Sridharan H, Ragan KB, Guo H, et al. Murine cytomegalovirus IE3-dependent transcription is required for DAI/ZBP1mediated necroptosis. EMBO Rep. 2017;18(8):1429-1441.

10. Upton JW, Kaiser WJ, Mocarski ES. DAI/ZBP1/DLM-1 complexes with RIP3 to mediate virus-induced programmed necrosis that is targeted by murine cytomegalovirus vIRA. Cell Host Microbe. 2012;11(3):290-297.

11. Newton K, Wickliffe KE, Maltzman A, et al. RIPK1 inhibits ZBP1-driven necroptosis during development. Nature. 2016; 540(7631):129-133

12. Lin J, Kumari S, Kim C, et al. RIPK1 counteracts ZBP1-mediated necroptosis to inhibit inflammation. Nature. 2016; 540(7631):124-128

13. Jiao H, Wachsmuth L, Kumari S, et al. Znucleic-acid sensing triggers ZBP1-dependent necroptosis and inflammation. Nature 2020:580(7803):391-395.

14. Tanaka Y, Chen ZJ. STING specifies IRF3 phosphorylation by TBK1 in the cytosolic DNA signaling pathway. Sci Signal. 2012;5(214):ra20

15. Ablasser A, Goldeck M, Cavlar T, et al. cGAS produces a $2^{\prime}-5^{\prime}$-linked cyclic dinucleotide second messenger that activates STING. Nature. 2013;498(7454):380-384

16. Mankan AK, Schmidt T, Chauhan D, et al. Cytosolic RNA:DNA hybrids activate the cGAS-STING axis. EMBO J. 2014; 33(24):2937-2946.

17. Takaoka A, Wang Z, Choi MK, et al. DAI
(DLM-1/ZBP1) is a cytosolic DNA sensor and an activator of innate immune response. Nature. 2007;448(7152):501-505

18. Ishii KJ, Kawagoe T, Koyama S, et al. TANK-binding kinase-1 delineates innate and adaptive immune responses to DNA vaccines. Nature. 2008;451(7179):725-729.

19. Yang D, Liang Y, Zhao S, et al. ZBP1 mediates interferon-induced necroptosis. Cell Mol Immunol. 2020;17:356-368.

20. Kuriakose T, Man SM, Malireddi RKS, et al. $\mathrm{ZBP} 1 / \mathrm{DAI}$ is an innate sensor of influenza virus triggering the NLRP3 inflammasome and programmed cell death pathways. Sci Immunol. 2016;1(2):aag2045.

21. Sarin V, Yu K, Ferguson ID, et al. Evaluating the efficacy of multiple myeloma cell lines as models for patient tumors via transcriptomic correlation analysis. Leukemia. 2020;34(10):2754-2765.

22. Dobin A, Davis CA, Schlesinger F, et al. STAR: ultrafast universal RNA-seq aligner. Bioinformatics. 2013;29(1):15-21.

23. Patro R, Duggal G, Love MI, Irizarry RA Kingsford C. Salmon provides fast and bias-aware quantification of transcript expression. Nat Methods. 2017;14(4):417419.

24. Love MI, Huber W, Anders S. Moderated estimation of fold change and dispersion for RNA-seq data with DESeq2. Genome Biol. 2014;15(12):550.

25. Ritchie ME, Phipson B, Wu D, et al. limma powers differential expression analyses for RNA-sequencing and microarray studies. Nucleic Acids Res 2015;43(7):e47.

26. Zhang Y, Liu T, Meyer CA, et al. Modelbased analysis of ChIP-Seq (MACS). Genome Biol. 2008;9(9):R137.

27. Ramírez F, Ryan DP, Grüning B, et al. deepTools2: a next generation web server for deep-sequencing data analysis. Nucleic Acids Res. 2016;44(W1):W160-165.

28. Thorvaldsdóttir H, Robinson JT, Mesirov P. Integrative Genomics Viewer (IGV) high-performance genomics data visualization and exploration. Brief Bioinform. 2013;14(2):178-192.

29. Heinz S, Benner C, Spann N, et al. Simple combinations of lineage-determining transcription factors prime cis-regulatory elements required for macrophage and $\mathrm{B}$ cell dentities. Mol Cell. 2010;38(4):576-589.

30. Wang S, Sun H, Ma J, et al. Target analysis by integration of transcriptome and ChIPdata with BETA. Nat Protoc. 2013;8(12):2502-2515.

31. Subramanian A, Tamayo P, Mootha VK, et al. Gene set enrichment analysis: a knowledge-based approach for interpreting genome-wide expression profiles. Proc Natl Acad Sci U S A. 2005;102(43):15545-15550.

32. Kuleshov MV, Jones MR, Rouillard AD, et al. Enrichr: a comprehensive gene set enrichment analysis web server 2016 update. Nucleic Acids Res. 2016;44(W1): W90-97.

33. Cattoretti G, Shaknovich R, Smith PM, Jäck H-M, Murty VV, Alobeid B. Stages of germinal center transit are defined by $B$ cell ranscription factor coexpression and relative abundance. J Immunol. 2006 177(10):6930-6939

34. Ochiai K, Maienschein-Cline M, Simonett $\mathrm{G}$, et al. Transcriptional regulation of ger- minal center B and plasma cell fates by dynamical control of IRF4. Immunity. 2013;38(5):918-929.

35. Buehler E, Chen Y-C, Martin S. C911: bench-level control for sequence specific siRNA off-target effects. PLoS One. 2012;7(12):e51942

36. Zuber J, McJunkin K, Fellmann C, et al. Toolkit for evaluating genes required fo proliferation and survival using tetracycline-regulated RNAi. Nat Biotechnol. 2011;29(1):79-83

37. Laganà A, Perumal D, Melnekoff D, et al. Integrative network analysis identifies novel drivers of pathogenesis and progres sion in newly diagnosed multiple myeloma. Leukemia. 2018;32(1):120-130.

38. Kuiper R, Broyl A, de Knegt Y, et al. A gene expression signature for high-risk multiple myeloma. Leukemia. 2012;26(11):2406 2413.

39. Muvaffak A, Pan Q, Yan H, et al. Evaluating TBK1 as a therapeutic target in cancers with activated IRF3. Mol Cancer Res. 2014;12(7):1055-1066.

40. Wang Z, Choi MK, Ban T, et al. Regulation of innate immune responses by DAI (DLM 1/ZBP1) and other DNA-sensing molecules. Proc Natl Acad Sci U S A. 2008, 105(14):5477-5482

41. Shaffer AL, Emre NCT, Lamy L, et al. IRF4 addiction in multiple myeloma. Nature. 2008;454(7201):226-231.

42. Zheng R, Wan C, Mei S, et al. Cistrome Data Browser: expanded datasets and new tools for gene regulatory analysis. Nucleic Acids Res. 2019;47(D1):D729-D735.

43. Jin Y, Chen K, De Paepe A, et al. Active enhancer and chromatin accessibility landscapes chart the regulatory network of primary multiple myeloma. Blood. 2018; 131(19):2138-2150

44. Kesavardhana S, Malireddi RKS, Burton $A R$, et al. The Z $\alpha 2$ domain of ZBP1 is a molecular switch regulating influenzainduced PANoptosis and perinatal lethality during development. I Biol Chem. 2020;295(24): 8325-8330.

45. Yang D, Liang Y, Zhao S, et al. ZBP1 mediates interferon-induced necroptosis. Cell Mol Immunol. 2020;17(4):356-368.

46. Hose $D$, Rème $T$, Hielscher $T$, et al. Proliferation is a central independent prognostic factor and target for personalized and risk-adapted treatment in multiple myeloma. Haematologica. 2011;96(1):87 95

47. Rebsamen M, Heinz LX, Meylan E, et al. DAI/ZBP1 recruits RIP1 and RIP3 through RIP homotypic interaction motifs to activate NF-kappaB. EMBO Rep. 2009; 10(8):916-922.

48. Tian W-L, Jiang Z-X, Wang F, et al. IRF3 is involved in human acute myeloid leukemia through regulating the expression of $\mathrm{miR}$ 155. Biochem Biophys Res Commun 2016;478(3):1130-1135

49. Pillai S, Nguyen J, Johnson J, Haura E Coppola D, Chellappan S. Tank binding kinase 1 is a centrosome-associated kinase necessary for microtubule dynamics and mitosis. Nat Commun. 2015;6(1):1-14.

50. Quinlan AR, Hall IM. BEDTools: a flexible suite of utilities for comparing genomic features. Bioinformatics. 2010;26(6):841-842. 